MADPH-98-1039

\title{
Constraints on Strong Dynamics from Rare B and K Decays ${ }^{1}$
}

\author{
Gustavo Burdman \\ Department of Physics, University of Wisconsin, Madison WI 53706.
}

\begin{abstract}
We discuss the constraints from rare B and $\mathrm{K}$ decays on the Electroweak Symmetry Breaking (EWSB) sector, as well as on theories of fermion masses. We focus on models involving new strong dynamics and show that transitions involving Flavor Changing Neutral Currents (FCNC) play an important role in disentangling the physics in these scenarios. In a model-independent approach to the EWSB sector, the information from rare decays is complementary to precision electroweak observables in bounding the contributions to the effective lagrangian. We compare the pattern of deviations from the Standard Model (SM) that results from these sources, with the deviations associated with the mechanism for generating fermion masses.
\end{abstract}

\section{INTRODUCTION}

Two of the most intriguing questions in particle physics are the the EWSB mechanism and the origin of fermion masses. Although the SM remains a successful theory when compared with all the available data, it lacks predictability in the Higgs sector, which determines the masses of gauge bosons, as well as of fermions through ad hoc Yukawa couplings. This suggests the possibility that new physics beyond the SM might be associated with either of these questions. In general, the energy scales and dynamics behind the EWSB sector and the fermion masses may be unrelated. In order to avoid fine-tuning, the scale associated with EWSB cannot be much higher than a few $\mathrm{TeV}$, whereas the scales where light fermion masses are generated could be much higher. If the mechanism responsible for the breaking of the electroweak symmetry involves some new strong dynamics, deviations from the SM might be observable in low energy signals even at energies much smaller than the scale of new physics $\Lambda$. Reaching this new frontier by direct observation of new physical states or even of tree-level effects in the couplings of SM particles, may require not only very large energies but also some previous knowledge of what (and what not) to expect. Thus, low energy measurements might be of paramount

1) Talk presented at the Workshop on Physics at the First Muon Collider and the Front End of the Muon Collider; November 6-9 1997, Fermilab, Batavia, Illinois. 
importance in planning experiments and search strategies at high energy machines. Among these low energy signals are electroweak measurements such as those at LEP and the Tevatron. On the other hand, processes involving Flavor Changing Neutral Currents (FCNC) can play a complementary role, since the fact that these processes are largely suppressed or forbidden in the SM may compensate the suppression by factors of $m / \Lambda$ (with $m$ the low energy scale, e.g. $m_{K}, m_{B}$, etc.). Here we address the potential of rare $B$ and $K$ decays as a complement to other low energy measurements in constraining models where strong dynamics is associated to either the EWSB sector and/or the origin of fermion masses. In the absence of a completely satisfactory theory of dynamical symmetry breaking and fermion masses, it is convenient to carry out a model-independent analysis that makes maximum use of the known properties of the electroweak interactions. This is the case with the EWSB sector, where an effective lagrangian approach allows us to parameterize the effects of the new strong dynamics in very much the same way chiral perturbation theory parameterizes low energy QCD. On the other hand, the effects from fermion mass generation can also be addressed by a general operator analysis. However, in addition, most theories predict the existence of relatively light states (scalars, pseudo-Goldstone bosons, etc.) which generally couple to mass in one way or another. To exemplify the effects of such states (which cannot be integrated out) we work with a particular set of models known as Topcolor-assisted Technicolor (TaTC). This provides a current example of how strong dynamics model building deals with the large top-quark mass and illustrates the distinct low energy phenomenology emerging from non-standard EWSB scenarios.

\section{LOW ENERGY EFFECTS OF ELECTROWEAK SYMMETRY BREAKING}

In the absence of a light Higgs boson the symmetry breaking sector is represented by a non-renormalizable effective lagrangian corresponding to the non-linear realization of the $\sigma$ model. The essential feature is the spontaneous breaking of the global symmetry $S U(2)_{L} \times S U(2)_{R} \rightarrow S U(2)_{V}$. To leading order the interactions involving the Goldstone bosons associated with this mechanism and the gauge fields are described by the effective lagrangian [1]

$$
\mathcal{L}_{L O}=-\frac{1}{4} B_{\mu \nu} B^{\mu \nu}-\frac{1}{2} \operatorname{Tr}\left[W_{\mu \nu} W^{\mu \nu}\right]+\frac{v^{2}}{4} \operatorname{Tr}\left[D_{\mu} U^{\dagger} D^{\mu} U\right]
$$

where $B_{\mu \nu}$ and $W_{\mu \nu}=\partial_{\mu} W_{\nu}-\partial_{\nu} W_{\mu}+i g\left[W_{\mu}, W_{\nu}\right]$ are the the $U(1)_{Y}$ and $S U(2)_{L}$ field strengths respectively, the electroweak scale is $v \simeq 246 \mathrm{GeV}$ and the Goldstone bosons enter through the matrices $U(x)=e^{i \pi(x)^{a} \tau_{a} / v}$. The covariant derivative acting on $U(x)$ is given by $D_{\mu} U(x)=\partial_{\mu} U(x)+i g W_{\mu}(x) U(x)-\frac{i}{2} g^{\prime} B_{\mu}(x) U(x) \tau_{3}$. To this order there are no free parameters once the gauge boson masses are fixed. The dependence on the dynamics underlying the strong symmetry breaking sector appears at next to leading order. To this order, a complete set of operators includes 
one operator of dimension two and nineteen operators of dimension four $[1,2]$. The effective lagrangian to next to leading order in the basis of Ref. [1] is given by

$$
\mathcal{L}_{\text {eff. }}=\mathcal{L}_{L O}+\sum_{i=0}^{19} \alpha_{i} \mathcal{O}_{i}
$$

where $\mathcal{O}_{0}$ is a dimension two custodial-symmetry violating term absent in the heavy Higgs limit of the SM. If we restrict ourselves to CP invariant structures, there remain fifteen operators of dimension four. The coefficients of some of these operators are constrained by low energy observables. For instance precision electroweak observables constrain the coefficient of $\mathcal{O}_{0}$, which gives a contribution to the electroweak parameter $T$. The $3 \sigma$ limit requires

$$
\alpha_{0}<6 \times 10^{-3}
$$

The combinations $\left(\alpha_{1}+\alpha_{8}\right)$ and $\left(\alpha_{1}+\alpha_{13}\right)$ contribute to the electroweak parameters $S$ and $U$. For instance, the constraint on $S$ translates into

$$
\left|\alpha_{1}+\alpha_{13}\right|<1.5 \times 10^{-2} .
$$

In addition, the coefficients $\alpha_{2}, \alpha_{3}, \alpha_{9}$ and $\alpha_{14}$ modify the triple gauge-boson couplings (TGC) and will be probed at LEPII and the Tevatron at the few percent level [3].

The remaining operators contribute to oblique corrections only to one loop and, in some cases, only starting at two loops. To the last group belong $\mathcal{O}_{11}$ and $\mathcal{O}_{12}$ given that their contributions to the gauge boson two-point functions only affect the longitudinal piece of the propagators. Of particular interest is the operator $\mathcal{O}_{11}$ defined by [1]

$$
\mathcal{O}_{11}=\operatorname{Tr}\left[\left(\mathcal{D}_{\mu} V^{\mu}\right)^{2}\right]
$$

with $V_{\mu}=\left(D_{\mu} U\right) U^{\dagger}$ and the covariant derivative acting on $V_{\mu}$ defined by $\mathcal{D}_{\mu} V_{\nu}=$ $\partial_{\mu} V_{\nu}+i g\left[W_{\mu}, V_{\nu}\right]$. The equations of motion for the $W_{\mu \nu}$ field strength imply [4]

$$
\mathcal{D}_{\mu} V^{\mu}=\frac{2 i}{v^{2}} \mathcal{D}_{\mu} J_{w}^{\mu}
$$

where the $S U(2)_{L}$ current is $J_{w}^{\mu}=\sum_{\psi}\left(\bar{\psi}_{L} \gamma^{\mu} \frac{\tau^{a}}{2} \psi_{L}\right) \tau^{a}, \psi_{L}$ denote the left-handed fermion doublets. The dominant effect appears in the quark sector due to the presence of terms proportional to $m_{t}$. After the quark fields are rotated to the mass eigenstate basis, the operator $\mathcal{O}_{11}$ can be written as [5]

$$
\mathcal{O}_{11}=\frac{m_{t}^{2}}{v^{4}}\left\{\left(\bar{t} \gamma_{5} t\right)^{2}-8 \sum_{i, j} V_{t i}^{*} V_{t j}\left(\bar{q}_{i_{L}} t_{R}\right)\left(\bar{t}_{R} q_{j L}\right)\right\}+\ldots
$$


where $i, j=d, s, b$, the $V_{t i}$ are Cabibbo-Kobayashi-Maskawa (CKM) matrix elements and the dots stand for terms suppressed by small fermion masses.

From the above discussion we see that the leading effects of the EWSB sector in FCNC processes are coming from the insertion of anomalous TGC vertices and four-fermion operators like (7). In the rest of this section, we review the status and future impact of these constraints on the symmetry breaking sector.

\section{Four-fermion Operators}

The effects of the four-fermion operators in (7) in rare $\mathrm{B}$ and $\mathrm{K}$ decays were considered in Ref. [6]. The loop insertion will result in contributions to several FCNC processes, that are controlled by both the coefficient $\alpha_{11}$ of the effective lagrangian (2) as well as by the high energy scale $\Lambda$. To one loop, only one parameter is needed, namely

$$
y=\alpha_{11} \log \frac{\Lambda^{2}}{m_{t}^{2}} .
$$

This parameter also governs the contributions of (7) to other neutral processes, both flavor changing and flavor conserving. For instance, the $\left(\bar{b}_{L} t_{R}\right)\left(\bar{t}_{R} b_{L}\right)$ term in (7) gives a contribution to $Z \rightarrow b \bar{b}$, whereas the terms like $\left(\bar{b}_{L} t_{R}\right)\left(\bar{t}_{R} d_{L}\right)$ appear in $B^{0}-\bar{B}^{0}$ mixing [5]. Thus the measurements of $R_{b}$ and the rate of $B$ mixing (together with all other CKM information) can be used to derive a bound on $y$. Although the bound carries some uncertainty mainly associated with CKM quantities like $f_{B}$ and $V_{u b}$, we will take it to be, approximately $[5,6]$

$$
|y|<0.50 \text {. }
$$

Next, we use this as the allowed range for $y$ in order to explore the possible impact of this physics in rare $B$ and $K$ decays. The one-loop insertion of the terms

$$
\mathcal{O}_{11}=-\frac{8 m_{t}^{2}}{v^{4}}\left\{V_{t s}^{*} V_{t b} \bar{s}_{L} t_{R} \bar{t}_{R} b_{L}+V_{t d}^{*} V_{t b} \bar{d}_{L} t_{R} \bar{t}_{R} b_{L}+V_{t d}^{*} V_{t s} \bar{d}_{L} t_{R} \bar{t}_{R} s_{L}\right\}+\ldots
$$

induces new contributions to various FCNC vertices in $B$ decays (the first two terms in (10)), as well as in $K$ decays (third term in (10)).

First, let us consider $b \rightarrow q \gamma$ processes leading, for instance, to the inclusive $B \rightarrow X_{s} \gamma$, since this rate has been recently measured [7]. The one-loop insertion of the operator $\mathcal{O}_{11}$ does not give a contribution to these processes given that it does not mix with the operator $\bar{s}_{L} \sigma_{\mu \nu} b_{R}$ responsible for the on-shell photon amplitude. Mixing only occurs at two loops, when QCD corrections are taken into account. As a result the effect, in all $b \rightarrow q \gamma$ transitions is expected to be only a few percent of the SM branching ratios [6].

On the other hand, the off-shell amplitudes for photons, $Z$ 's and gluons are nonzero at one loop. They generate contributions to processes such as $b \rightarrow q \ell^{+} \ell^{-}$, 


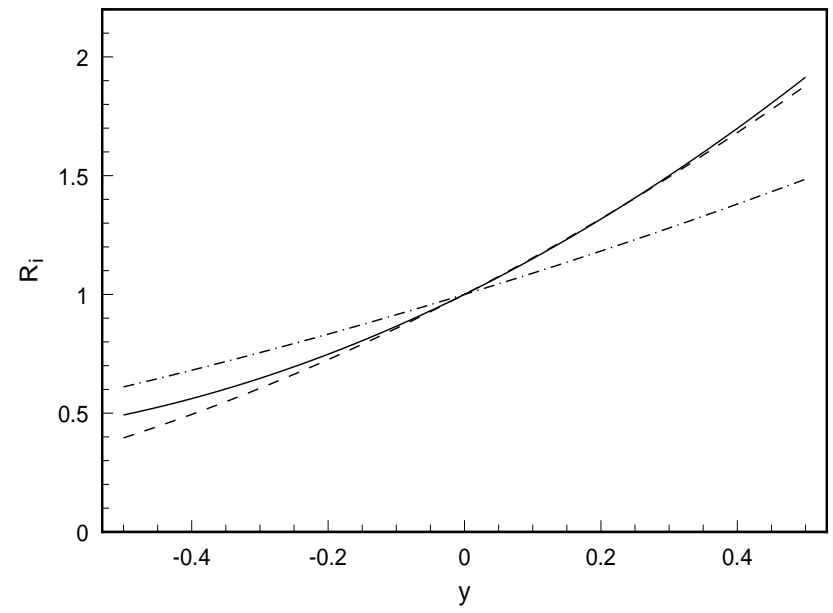

FIGURE 1. Ratio of the modified branching ratio to the standard model expectation as a function of $y=\alpha_{11} \log \frac{\Lambda^{2}}{m_{t}^{2}}$. The solid line corresponds to the ratio $R_{\ell}$ for $B \rightarrow X_{(s, d)} \ell^{+} \ell^{-}$ inclusive decays, the dashed line to $R_{\nu}$ for $B \rightarrow X_{(s, d)} \nu \bar{\nu}$ and the dot-dashed line to $R_{g}$ for $b \rightarrow s \bar{s} s$ decays. From Ref. [6].

$b \rightarrow q \nu \bar{\nu}, b \rightarrow q \bar{q}^{\prime} q^{\prime}$; as well as to similar rare kaon decays like $s \rightarrow d \nu \bar{\nu}$, etc. In order to asses the potential effects we define

$$
R_{\ell} \equiv \frac{\operatorname{Br}\left(B \rightarrow X_{(s, d)} \ell^{+} \ell^{-}\right)}{\operatorname{Br}\left(B \rightarrow X_{(s, d)} \ell^{+} \ell^{-}\right)_{\mathrm{SM}}},
$$

which is plotted in Fig. 1 as a function of the parameter $y$ defined in (8), for the allowed range of $y(9)$. Analogously, we can define the ratio $R_{\nu}$, which tracks the effects in $B \rightarrow X_{s, d} \nu \bar{\nu}$ decays; whereas the contribution to gluon penguin processes such as $b \rightarrow s \bar{s} s$ is represented by the ratio $R_{g}$. As it is clear from Fig. 1, the effects of the operator $\mathcal{O}_{11}$ are very similar in all three types of $B$ decays.

We see that, even with the $R_{b}$ and $B^{0}-\bar{B}^{0}$ mixing constraints, large deviations from the SM predictions for these modes are possible. The current experimental bounds on these processes are still not binding on $y$. However, sensitivity to SM branching ratios will be reached in the next round of experiments at the various $B$ factories at Cornell, KEK, SLAC and Fermilab. The distinct feature of this effect is that no significant deviation is expected in $b \rightarrow s \gamma$, even when large deviations are observed in all the other modes.

The effects are very similar in rare $K$ decays such as $K^{+} \rightarrow \pi^{+} \nu \bar{\nu}$ and $K_{L} \rightarrow$ $\pi^{0} \nu \bar{\nu}$, etc. In Fig. 2 we plot $R_{K}$, a quantity analogous to $R_{\ell}$ in (11). Again, large effects of up to factors of 2 deviations, are allowed. The recently reported [8] observation of one event in $K^{+} \rightarrow \pi^{+} \nu \bar{\nu}$ roughly translates into $R_{K} \simeq(0.50-5.0)$, which is still not constraining.

Although in this model-independent approach we cannot, as a matter of principle, calculate the size of the coefficients $\alpha_{i}$, we can use general arguments to estimate their approximate value. Using naive dimensional analysis [9] we have 


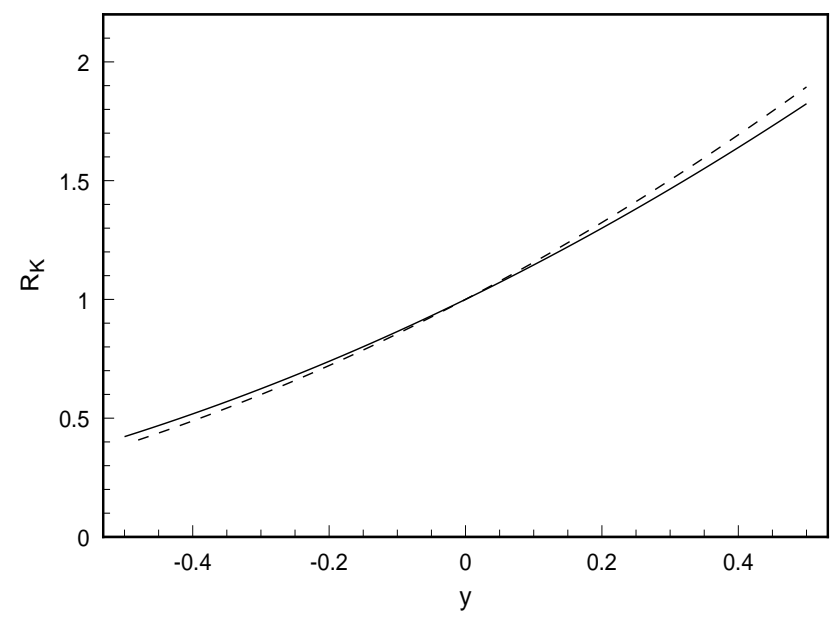

FIGURE 2. Ratio of the modified branching ratio to the standard model expectation for $K^{+} \rightarrow \pi^{+} \nu \bar{\nu}$ (solid line) and $K_{L} \rightarrow \pi^{0} \nu \bar{\nu}$ (dashed line). From Ref. [6].

$$
\alpha_{11} \simeq \mathcal{O}(1) \times \frac{v^{2}}{\Lambda^{2}}
$$

with the scale of new physics obeying $\Lambda \lesssim 4 \pi v$. For instance, taking $\Lambda=4 \pi v$, one would obtain $y \simeq \mathcal{O}(1) \times 0.04$. On the other hand, if $\Lambda=2 \pi v$, one has $y \simeq \mathcal{O}(1) \times 0.12$. In any case, these are meant to be order of magnitude estimates. Therefore, the experimental relevance of the effect strongly depends on details of the dynamics we are not able to compute in a model-independent fashion.

Finally, we should note that rare $B$ and $K$ decays are the most sensitive signals for this effect. This is due to the fact that four-lepton operators are suppressed by the lepton masses, and that $\mathcal{O}_{11}$ does not mix quarks and leptons. 


\section{Triple Gauge-boson Couplings}

Imposing $C$ and $P$ conservation, the most general form of the $W W N(N=\gamma, Z)$ couplings can be written as [3]

$$
\begin{aligned}
\mathcal{L}_{W W N}= & g_{W W N}\left\{i \kappa_{N} W_{\mu}^{\dagger} W_{\nu} N^{\mu \nu}+i g_{1}^{N}\left(W_{\mu \nu}^{\dagger} W^{\mu} N^{\nu}-W_{\mu \nu} W^{\dagger \mu} N^{\nu}\right)\right. \\
& \left.+i \frac{\lambda_{N}}{M_{W}^{2}} W_{\mu \nu}^{\dagger} W_{\lambda}^{\nu} N^{\nu \lambda}\right\},
\end{aligned}
$$

with the conventional choices being $g_{W W \gamma}=-e$ and $g_{W W Z}=-g \cos \theta$ [10]. In principle, there are six free parameters. Making contact with the electroweak lagrangian (2), these parameters can be expressed in terms of the next-to-leading order coefficients [4] $\alpha_{1}, \alpha_{2}, \alpha_{3}, \alpha_{8}, \alpha_{9}, \alpha_{13}$ and $\alpha_{14}$. Conservation of the electromagnetic charge implies $g_{1}^{\gamma}=1$. Furthermore, to this order in the energy expansion (2) $\lambda_{N}=0$. Then we are left with $\kappa_{\gamma}, \kappa_{Z}$ and $g_{1}^{Z}$. Finally, when considering rare $B$ and $K$ decays, we can neglect the contribution of $\kappa_{Z}$ since it will be suppressed by powers of the small external momenta over $m_{Z}$. Thus, in this simplistic approach, there are only two parameters relevant at very low energies. The SM predicts $\kappa_{\gamma}=g_{1}^{Z}=1$. The effects of anomalous TGC have been previously studied in the literature [11]. However, this hierarchical approach to the couplings has not been the one used in the various analyses and a more comprehensive study is needed. The experiments at LEP II and the next Tevatron run are going to be sensitive to deviations from the SM prediction at the $(5-10) \%$ level [10]. Effects of this size

might be also observed in rare $B$ and $K$ decays. For instance, $\delta g_{1}^{Z}=g_{1}^{Z}-1=0.10$ can produce enhancements in the branching ratios of $b \rightarrow s \ell^{+} \ell^{-}$decay modes of up to $(60-70) \%$ [11]. In the near future, $B$ factory experiments will have sensitivity to these processes at the SM level, turning these low energy measurements into an excellent complement of direct probes of the TGC.

\section{FERMION MASSES AND ELECTROWEAK DYNAMICS}

Up to now, we have only considered the effects of the dynamics associated with the EWSB. These are encoded in the effective lagrangian (2), which only involves the Goldstone boson and gauge fields. Additionally, it is possible that the new strong dynamics may also affect some or all fermions. We first comment on the effective lagrangian approach for non-SM couplings of fermions to gauge bosons, and then examine the effects of a prototypical class of theories (Topcolor) where the dynamical generation of fermion masses imply the existence of relatively light new states.

\section{Anomalous Couplings of Fermions to Gauge Bosons}

The effects of new dynamics on the couplings of fermions with the SM gauge bosons can be, in principle, also studied in an effective lagrangian approach. For 
instance, if in analogy with the situation in QCD, fermion masses are dynamically generated in association with EWSB, residual interactions of fermions with Goldstone bosons could be important [12] if the $m_{f} \simeq f_{\pi} \simeq v$. Thus residual, nonuniversal interactions of the third generation quarks with gauge bosons could carry interesting information about both the origin of the top quark mass and EWSB. In a very general parameterization, the anomalous couplings of third generation quarks can be written as

$$
\begin{aligned}
\Delta \mathcal{L}= & -\frac{g}{\sqrt{2}}\left\{C_{L}\left(\bar{t}_{L} \gamma_{\mu} b_{L}\right)+C_{R}\left(\bar{t}_{R} \gamma_{\mu} b_{R}\right)\right\} W^{+\mu} \\
& -\frac{g}{2 c \theta_{W}}\left\{N_{L}^{t}\left(\bar{t}_{L} \gamma_{\mu} t_{L}\right)+N_{R}^{t}\left(\bar{t}_{R} \gamma_{\mu} t_{R}\right)\right. \\
& \left.+N_{L}^{b}\left(\bar{b}_{L} \gamma_{\mu} b_{L}\right)+N_{R}^{b}\left(\bar{b}_{R} \gamma_{\mu} b_{R}\right)\right\} Z^{\mu}
\end{aligned}
$$

where the parameters $C_{L, R}, N_{L, R}^{t, b}$ contain the residual, non-universal effects associated with the new dynamics, perhaps responsible for the large top quark mass. Then, if we assume that the new couplings are CP conserving, there are six new parameters. They are constrained at low energies by a variety of experimental information, mostly from electroweak precision measurements and the rate of $b \rightarrow s \gamma$. Several simplifications are usually made in order to reduce the number of free parameters. For instance, in most of the literature, it is assumed that $N_{L, R}^{b}=0$ [14]. A stringent bound on the right-handed charged coupling is obtained from $b \rightarrow s \gamma$ [15]: $-0.05<C_{R}<0.01$. The bounds obtained on a particular coupling from electroweak observables such as $S, T, U$ and $R_{b}$ generally strongly depend on assumptions about the other couplings. For example, if $C_{L}=0$, then the combination $\left(N_{L}^{t}-N_{R}^{t}\right)$ is strongly constrained since it contributes to $T$. On the other hand, if $C_{L}=N_{L}^{t}$, then $N_{R}^{t}<0.02[12,16]$ since it is the only (linear) contribution to $T$. Thus, although in general most parameters are confined to a few percent, some of them are allowed to be as large as 0.30 under certain conditions. This "modeldependent" situation requires more experimental information. A global analysis of the effects of the couplings of eqn. (15) in rare $B$ and $K$ processes such as $b \rightarrow s \ell^{+} \ell^{-}, s \rightarrow d \nu \bar{\nu}$, etc. may help disentangle the various possible effects and perhaps will give constraints that may be of importance in interpreting data from higher energy experiments [17].

\section{The effects of light states: the example of Topcolor}

The description of the residual effects of strong dynamics at low energies on fermion couplings by using (15) corresponds to cases where the states associated with the new physics are heavy compared to the weak scale. Thus, integrating out the heavy states, leaves us with effective couplings which might be generated at tree level or through loops in the full theory. However, most theories in which electroweak symmetry and/or fermion masses have a dynamical origin also contain 
states with masses comparable to the weak scale. Such is the case, for instance, in Technicolor models where the breaking of large chiral symmetries imply the presence of pseudo-Goldstone bosons with masses of at most a few hundred GeV . It is also the case in Topcolor-assisted Technicolor (TaTC) models [18], where a top-condensation mechanism generated by the Topcolor interactions is responsible for the large dynamical top quark mass, whereas Technicolor breaks the electroweak symmetry giving (most of) the $W$ and $Z$ masses. The TaTC scenario is designed to relief the problems of Extended Technicolor (ETC) in generating a heavy top [19]. Although the new gauge bosons associated with the TaTC gauge group are heavier than $1 \mathrm{TeV}$, the presence of several scalar and pseudo-scalar states with masses in the few-hundred $\mathrm{GeV}$ range, forces us to take these into account directly in our calculations. From the point of view of their impact in low energy observables, the most important of these states are the top-pions $\vec{\pi}_{t}$, the triplet of Goldstone bosons associated with the breaking of the top chiral symmetry. Since top condensation does not fully break the electroweak symmetry $\left(f_{\pi_{t}} \simeq(60-70) \mathrm{GeV}<v\right)$, after mixing with the techni-pions, there will be a triplet of physical top-pions in the spectrum, with a coupling to third generation quarks given by

$$
i \frac{m_{t}}{\sqrt{2} f_{\pi_{t}}}\left\{\bar{t} \gamma_{5} t \pi^{0}+\bar{t}_{R} b_{L} \pi^{+}+\bar{b}_{L} t_{R} \pi^{-}\right\} \text {. }
$$

They acquire masses of a few hundred GeV due to explicit ETC quark mass terms. Additionally, in most models there are scalar and pseudo-scalar bound states due to the strong (although sub-critical) effective coupling of right-handed $b$-quarks. The closer the effective couplings are from criticality, the lighter these bound states tend to be. The spectrum and properties of these states, unlike those of top-pions, are not determined by model-independent features of the symmetry breaking pattern but depend on details of the model. Finally, in all TaTC models there will be pseudo-Goldstone bosons from the breaking of techni-fermion chiral symmetries. However, their couplings to third generation quarks are reduced with respect to (16) by $m_{E T C} / m_{t}$, where $m_{E T C}$ is a small ETC mass of the order of $1 \mathrm{GeV}^{2}$. The presence of the relatively light top-pions, as well as the additional bound states, imposes severe constraints on Topcolor models due to their potential loop effects in low energy observables, most notably $R_{b}$ and rare $B$ and $K$ decays.

Top-pion Effects in $R_{b}$ : The one-loop contributions of top-pions to the $Z \rightarrow \bar{b} b$ process were studied in Ref. [21]. There it was shown that they shift $R_{b}$ negatively by an amount controlled by $m_{\pi_{t}}$ and $f_{\pi_{t}}$. For instance, for $f_{\pi_{t}} \simeq 60 \mathrm{GeV}$ the correction is about $-1 \%$ for $m_{\pi_{t}}=800 \mathrm{GeV}$, and top-pions with masses in the expected $(100-300) \mathrm{GeV}$ range give unacceptably large deviations. This value of the top-pion decay constant is obtained by using the Pagels-Stokar formula, which gives $f_{\pi_{t}}$ a logarithmic dependence on the Topcolor energy scale, chosen here to be

2) Multi-scale Technicolor models such as the one in Ref. [20], in the absence of Topcolor, have un-suppressed top couplings to pseudo-scalars. This could lead to effects similar to those of top-pions. 


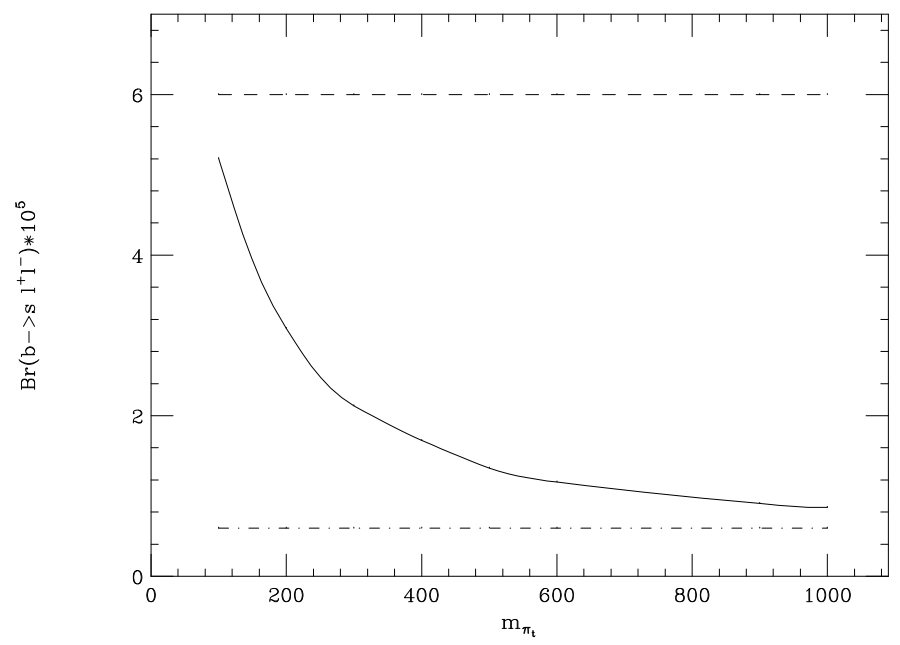

FIGURE 3. $\operatorname{Br}\left(b \rightarrow s \ell^{+} \ell^{-}\right)$vs. $m_{\pi_{t}}$, for $f_{\pi_{t}}=70 \mathrm{GeV}$. The dashed horizontal line is the current experimental limit for the inclusive rate [24], whereas the dot-dash line is the SM expectation.

a few TeV. Potentially cancelling contributions by other states, such as the scalar and pseudo-scalar bound states, Topcolor vector and axial-vector mesons, etc., are either of the wrong sign or not large enough. Possible ways out of this constraint are: larger top-pion masses or larger values of $f_{\pi_{t}}$. The larger $f_{\pi_{t}}$ is, the smaller the coupling, and the top-pions are more Goldstone-boson-like. For $f_{\pi_{t}} \simeq 120 \mathrm{GeV}$, for instance, the shift of $R_{b}$ is well within the experimentally allowed region even for $m_{\pi_{t}} \simeq(200-300) \mathrm{GeV}$. However, in order to obtain such an enhancement in the decay constant we must either assume large corrections to the Pagels-Stokar expression or introduce new and exotic fermion states.

Rare $B$ and $K$ Decays: The top-pions and other scalar states, give oneloop contributions to FCNC processes. These depend not only on $f_{\pi_{t}}$ and $m_{\pi_{t}}$ but typically also on one or more elements of the quark rotation matrices necessary to diagonalize the quark Yukawa couplings. The contributions of top-pions, as well as "b-pions" (scalar and pseudo-scalar bound states in models where $b_{R}$ couples to the Topcolor interaction) to $b \rightarrow s \gamma$ depend on $D_{L, R}^{b s}$, the $b \rightarrow s$ element in the left or right down rotation matrix. Furthermore, the two contributions tend to cancel. Thus, the freedom in this model-dependent aspects of the prediction makes it possible to have quite low masses and still satisfy the bound from the experimental measurement of $B \rightarrow X_{s} \gamma$ [22]. The situation changes drastically in $b \rightarrow s \ell^{+} \ell^{-}$processes, where the cancellations are much less efficient. Although experiments have not yet reached sensitivity to SM branching ratios [23], it will be soon achieved at both hadron and lepton $B$ factories. As an example, we plot in Fig. 3 the $\operatorname{Br}\left(b \rightarrow s \ell^{+} \ell^{-}\right)$as a function of the top-pion mass with no other contributions, for $f_{\pi_{t}}=70 \mathrm{GeV}$. The $b \rightarrow s \gamma$ constraint is in this case $\left(200 \mathrm{GeV}<m_{\pi_{t}}<800 \mathrm{GeV}\right)$. However, one can see that, even for heavier toppions the effect can still be a $(30-60) \%$ enhancement over the SM prediction of 
$6 \times 10^{-6}$. On the other hand, in the presence of a $400 \mathrm{GeV}$ charged b-pion the curve changes little, but the $b \rightarrow s \gamma$ bound is now $m_{\pi_{t}}<600 \mathrm{GeV}$. Finally, to compare the potential of these FCNC transitions with the $R_{b}$ constraints, let us say that if we take $f_{\pi_{t}} \simeq 120 \mathrm{GeV}$ (which avoids conflict with $R_{b}$ measurements), then the effect of a $400 \mathrm{GeV}$ top-pion in $b \rightarrow s \ell^{+} \ell^{-}$is still an enhancement of more than $50 \%$ with respect to $\mathrm{SM}$ expectations. Thus, the observation of these modes will further constrain Topcolor models beyond the $R_{b}$ bounds. We expect similar effects due to top-pions and/or b-pions to be present in kaon processes such as $K^{+} \rightarrow \pi^{+} \nu \bar{\nu}$.

\section{CONCLUSIONS}

We have seen that a complete, model-independent analysis of the effects of strong dynamics in rare $B$ and $K$ decays could shed light on the nature of the EWSB mechanism and the origin of fermion masses. The signals are also likely to be important in models where relatively light scalars couple strongly to mass, like in the case of TaTC. In most cases, the next round of experiments will have sensitivity to SM branching ratios. This will be the case, for instance, for the Tevatron experiments, as well the KEK and SLAC $B$ factories in the $B \rightarrow X_{(s, d)} \ell^{+} \ell^{-}$modes. It will also be the situation in the next generation of kaon experiments for $K^{+} \rightarrow$ $\pi^{+} \nu \bar{\nu}$ and $K_{L} \rightarrow \pi^{0} \nu \bar{\nu}$. The amount and variety of experimental information from these processes is such that suggests a parallel to the role of electroweak measurements at the $Z$ pole as not only a constraint on new physics sources but also as guidance in the searches to be carried out at high energy machines such as the Tevatron in Run II, the LHC and eventually the NLC and/or the muon collider. It is possible to imagine a scenario where deviations from the $\mathrm{SM}$ in $B$ and/or $K$ decays point to a particular source, e.g. corrections to Goldstone boson propagators given by $\mathcal{O}_{11}$, anomalous TGC or anomalous couplings of third generation quarks to gauge bosons as in (15). The nature of the deviation might dictate the road to follow at high energies. As an example, if the source of an effect is in one the top quark couplings $N_{L, R}^{t}$, there would be a strong case for a lepton collider running at $t \bar{t}$ threshold. Other scenarios may not be so clear, and may require a comprehensive and careful analysis of all the data to come (including issues like hadronic uncertainties in $B$ decays). This, however, constitutes a very well defined research program.

\section{REFERENCES}

1. A. Longhitano, Phys. Rev. D22, 1166 (1980), Nucl. Phys. B188, 118 (1981).

2. T. Appelquist and G. Wu, Phys. Rev. D48, 3235 (1993).

3. K. Hagiwara, K. Hikasa, R. D. Peccei and D. Zeppenfeld, Nucl. Phys. B282, 253 (1987); K. Hagiwara, S. Ishiara, R. Szalapski and D. Zeppenfeld, Phys. Lett. B283, 353 (1992) and Phys. Rev. D48, 2182 (1993).

4. F. Feruglio, Int. J. Mod. Phys. A8, 4937 (1993). 
5. J. Bernabéu, D. Comelli, A. Pich and A. Santamaria, Phys. Rev. Lett. 78, 2902 (1997).

6. G. Burdman, Phys. Lett. B409, 443 (1997).

7. R. Balest et al., the CLEO collaboration, Phys. Rev. Lett. 74, 2885 (1995).

8. S. Adler et al., the BNL 787 Collaboration, Phys. Rev. Lett. 79, 2204 (1997).

9. A. Manohar and H. Georgi, Nucl. Phys. B234, 189 (1984); H. Georgi, "Weak Interactions and Modern Particle Theory", Benjamin/Cummings, Menlo Park, California, 1984.

10. T. Barklow et al., SLAC-PUB-7366, In the Proceedings of "New Directions for HighEnergy Physics (Snowmass 96), Snowmass, CO, 25 Jun - 12 Jul 1996.

11. S. P. Chia, Phys. Lett. B240, 465 (1990); K. A. Peterson, Phys. Lett. B282, 207 (1992); G. Baillie, Z. Phys. C61, 667 (1994).

12. R. D. Peccei and X. Zhang, Nucl. Phys. B337, 269 (1990); R. D. Peccei, S. Peris and X. Zhang, Nucl. Phys. B349, 305 (1991).

13. For a treatment of $\mathrm{CP}$ violating effects in $B$ decays from these couplings see $\mathrm{A}$. Abd El-Hady and G. Valencia, Phys. Lett. B414, 173 (1997).

14. E. Malkawi and C. P. Yuan, Phys. Rev. D50, 4462 (1994).

15. J. Hewett and T. Rizzo, Phys. Rev. D49, 319 (1994); K. Fujikawa and A. Yamada, Phys. Rev. D49, 5890 (1994);

16. B. Dobrescu and J. Terning, Phys. Lett. B416, 129 (1998).

17. G. Burdman, in preparation.

18. C. T. Hill, Phys. Lett. B345, 483 (1995).

19. E. Eichten and K. Lane, Phys. Lett. B352, 382 (1995).

20. E. Eichten and K. Lane, Phys. Lett. B222, 274 (1989); ibid, 388, 803 (1996).

21. G. Burdman and D. Kominis, Phys. Lett. B403, 101 (1997).

22. G. Buchalla, G. Burdman, C. T. Hill and D. Kominis, Phys. Rev. D53, 5185 (1996).

23. C. Albajar et al., the UA1 collaboration, Phys. Lett. B262, 163 (1991); S. Glenn et al., the CLEO collaboration, CLNS-97/1514, hep-ex/9710003; B. Abbot et al., the D0 collaboration, hep-ex/9801027. 